\title{
Special issues on moment methods in kinetic gas theory
}

Received: 12 November 2009 / Accepted: 13 November 2009

(C) Springer-Verlag 2009

This two-volume special issue of 'Continuum Mechanics and Thermodynamics' is the result of an international meeting on moment methods in kinetic gas theory that took place in November 2008 at ETH Zurich [1]. There have been over 30 participants from 10 different countries attending 13 invited lectures on various developments in moment methods. The workshop was hosted jointly by the 'Research Institute for Mathematics (FIM)' and the 'Seminar for Applied Mathematics (SAM)' at ETH Zurich, as well as supported by the 'Swiss National Science Foundation (SNF)'.

In recent years, moment methods in kinetic gas theory proved to be a successful tool to extend classical fluid dynamics for applications in micro- or rarefied gas flows. The classical equations of compressible fluid dynamics, known as the system of Navier-Stokes and Fourier, loose their validity in extreme physical processes involving rarefaction or micro-scales. The lack of sufficient particle collisions is expressed in larger values of the Knudsen number $K n$-the ratio between the mean free path and an observation scale-and leads to thermal non-equilibrium which can only be described using refined mathematical models.

Kinetic gas theory based on a statistical description of the gas provides a valid framework to model processes in a rarefied regime or at small scales [2,3]. The main variable used to describe the gas is the distribution function or probability density of the particle velocities. However, in situations of intermediate non-equilibrium this detailed statistical approach still yields a far too complex description of the gas. In these situations it is desirable to have a continuum model based on partial differential equations for the fluid mechanical field variables.

The classical fluid dynamics equations can be derived from Boltzmann's equation as the first order contribution in a Chapman-Enskog expansion [4], an asymptotic expansion in the Knudsen number. It seemed natural to look for higher order contributions as an extension for the laws of Navier-Stokes and Fourier. However, the hope for useful equations was dashed by the observation of Bobylev [5] stating that these higher order models are inherently unstable in the general case.

In the 1940s Harold Grad [6,7] developed an alternative to the Chapman-Enskog expansion by producing moment equations from Boltzmann's equation. The fundamental idea of moment approximations is to replace the variable of a high dimensional distribution function $f(\mathbf{x}, t, \mathbf{c})$ for the velocity $\mathbf{c} \in \mathbb{R}^{3}$ by a finite set moment variables $\left\{F_{n}(\mathbf{x}, t)\right\}_{n=0,1,2, \ldots}$ at each $(\mathbf{x}, t)$. The working hypothesis is that at $(\mathbf{x}, t)$ a finite number of moments are sufficient to encode the details of $f$ as a function of $\mathbf{c}$. The first moments are the conservative variables of gas dynamics, the density, the momentum density and total energy density. Obviously, the motivation is that

Communicated by M. Torrilhon

M. Torrilhon $(\varangle)$

Seminar for Applied Mathematics, ETH, Zurich, Switzerland

E-mail: matorril@math.ethz.ch

Present address:

M. Torrilhon

Department of Applied Mathematics, University of Washington, Seattle, USA 
classical gas dynamics is very successful by describing the gas with very few moments. More moments should give an even better approximation of $f$, thus better describing non-equilibrium processes.

Moment equations in kinetic gas theory have seen a turbulent life. After being discarded by Grad himself due to bad performance, they were later accused of many other deficiencies, often wrongly. Popular objections are (see e.g., [8]): complexity of the equations, no relation to the Knudsen number, the need of many moments for strong non-equilibrium, lack of boundary conditions, lack of global hyperbolicity, prediction of non-physical sub-shocks, lack of thermodynamic backing by an entropy law. However, especially in recent years most of the objections could be pushed aside by refined modeling and a better understanding of the nature of moment equations.

Using many different applications, the work of Ingo Müller and his colleagues [9] demonstrates that moment equations are a capable tool to predict non-equilibrium processes if sufficiently many moments are considered. They also demonstrate a close link between moment equations and the constitutive theory of thermodynamics based on the exploitation of the entropy law. Levermore [10] gave a partial answer to the question of hyperbolicity of larger moment equations. Many other works exist considering computational and analytical investigations of moment equations, see e.g., [11-14] and the references in the papers of this issue.

In order to improve the accuracy of equations using only few moments several attempts tried to combine the approaches of Grad with the expansion of Chapman-Enskog [15,16]. The work in [17] presents the regularized 13-moment equations directly derived from Boltzmann's equation, which finally overcome the sub-shock problem, maintain stability and strongly increase the accuracy of the classical 13-moment-equations. Finally, boundary conditions for moment equations had been originally given already by Grad and pioneered by $\mathrm{Gu}$ and Emerson [18] for regularized moment equations. By now these have been further refined so that it is now possible to write down well-posed boundary value problems for (linear) moment equations as shown in the papers of this issue.

In my opinion there are two important facts which are often neglected when estimating the advantages of moment equations. When compared to natural alternatives for simulating non-equilibrium flows, e.g., direct Boltzmann solvers or Monte-Carlo simulations, one has to keep in mind that a 13-moment-system describes the state of the gas by using only 13 real field variables in each space point. This contrasts direct discretizations of Boltzmann's equation which very often use $10^{4}$ and more points to represent the distribution function, hence the state of the gas, in each space point. In a way, the values of the 13 moments encode the distribution function in a highly efficient compression. Furthermore - and this is the second fact-moment equations come as partial differential equations based on fluid dynamic variables like velocity and stress tensor. As such, they allow analytical solutions to be found and investigated leading to an increase in intuition based on mathematical insight, see various papers of this issue. In a time where computational models become increasingly complex but the results do not necessarily lead to a better actual understanding of the process, I consider this feature invaluable.

The papers in this and the following volume demonstrate impressively what can be achieved by moment methods and at the same time tackle the remaining open problems. Boundary value problems are investigated analytically in the papers of Gu et al. [19] and Taheri et al. [20], for regularized moment equations, as well as for the full moment hierarchy by Santos [21]. The papers of Groth and McDonald [22] and Suzuki et al. [23] discuss computational aspects of moment equations, while special variants of the moment method are investigated by Myong [24] and Frezzotti et al. [25]. In the papers by Bennoune et al. [26] and Seibold and Frank [27], this issue reaches out to hybrid kinetic discretizations and modeling of radiation, two fields with direct relations and impact to moment methods.

The special issues were made possible through the support of the journal 'Continuum Mechanics and Thermodynamics', especially Editor-in-Chief Stefan Seelecke, and Managing Editor Wolfgang Müller, to whom

I would like to express my gratitude also in the name of all authors. Special thanks go to my colleague Kun $\mathrm{Xu}$ from Hong Kong UST for bringing up the original idea of a meeting on moment methods.

\section{References}

1. International Workshop on Moment Methods in Kinetic Gas Theory, 6-8 November 2008, ETH Zurich, Switzerland, see www.math.ethz.ch/ matorril/Workshop

2. Cercignani, C.: The Boltzmann Equation and its Applications, Applied Mathematical Sciences 67. Springer, New York (1988)

3. Karniadakis, G.E., Beskok, A.: Micro Flows: Fundamentals and Simulation. Springer, New York (2001) 
4. Chapman, S., Cowling, T.G.: The Mathematical Theory of Non-Uniform Gases. Cambridge University Press, Cambridge (1970)

5. Bobylev, A.V.: The Chapman-Enskog and Grad methods for solving the Boltzmann equation. Sov. Phys. Dokl. 27, 29-31 (1982)

6. Grad, H.: On the kinetic theory of rarefied gases. Commun. Pure Appl. Math. 2, 331 (1949)

7. Grad, H.: Principles of the kinetic theory of gases. In: Flügge, S. (ed.) Handbuch der Physik, vol. 12. Springer, Berlin (1958)

8. Struchtrup, H.: Macroscopic Transport Equations for Rarefied Gas Flows. Springer, New York (2005)

9. Müller, I., Ruggeri, T.: Rational Extended Thermodynamics (2nd edn) Springer Tracts in Natural Philosophy (vol. 37). Springer, New York (1998)

10. Levermore, C.D.: Moment closure hierarchies for kinetic theories. J. Stat. Phys. 83(5-6), 1021-1065 (1996)

11. Eu, B.-C.: A modified moment method and irreversible themrodynamics. J. Chem. Phys. 73(6), 2958-2969 (1980)

12. Seeger, S., Hoffmann, H.: The comulant method in computational kinetic theory. Continuum Mech. Thermodyn. 12(6), 403 (2000)

13. Marquez, W. Jr., Kremer, G.M.: Couette flow from a 13 field theory with jump and slip boundary conditions. Continuum Mech. Thermdyn. 13, 104-117 (2001)

14. Vereshchagin, D.A., Leble, S.B., Solovchuk, M.A.: Piecewise continuous distribution function method in the theory of wave disturbances of inhomogeneous gas. Phys. Lett. A 348(3-6), 326-334 (2006)

15. Karlin, I.V., Gorban, A.N., Dukek, G., Nonnenmacher, T.F.: Dynamic correction to moment approximations. Phys. Rev. E 57(2), 1668-1672 (1998)

16. Jin, S., Slemrod, M.: Regularization of the Burnett equations via relaxation. J. Stat. Phys. 103(5-6), 1009-1033 (2001)

17. Struchtrup, H., Torrilhon, M.: Regularization of Grad's 13-moment-equations: derivation and linear analysis. Phys. Fluids 15(9), 2668-2680 (2003)

18. Gu, X.-J., Emerson, D.: A computational strategy for the regularized 13 moment equations with enhanced wall-boundary Conditions. J. Comput. Phys. 225, 263-283 (2007)

19. Gu, X.-J., Emerson, D.R., Tang, G.-H.: Kramers' problem and the Knudsen minimum: a theoretical analysis using a linearized 26-moment approach. Continuum Mech. Thermodyn. doi:10.1007/s00161-009-0121-5

20. Taheri, P., Rana, A.S., Torrilhon, M., Struchtrup, H.: Macroscopic description of steady and unsteady rarefaction effects in boundary value problems of gas dynamics. Continuum Mech.Thermodyn. doi:10.1007/s00161-009-0115-3

21. Santos, A.: Solutions of the moment hierarchy in the kinetic theory of Maxwell models. Continuum Mech.Thermodyn. doi:10.1007/s00161-009-0113-5

22. Groth, C.P.T., McDonald, J.G.: Towards physically realizable and hyperbolic moment closures for kinetic theory. Continuum Mech. Thermodyn. doi:10.1007/s00161-009-0125-1

23. Suzuki, Y., Khieu, L., van Leer, B.: CFD by first order PDE's. Continuum Mech. Thermodyn. doi:10.1007/s00161-0090124-2

24. Myong, R.S.: Coupled nonlinear constitutive models for rarefied and microscale gas flows: subtle interplay of kinematics and dissipation effects. Continuum Mech. Thermodyn. doi:10.1007/s00161-009-0112-6

25. Frezzotti, A., Gibelli, L., Franzelli, B.: A moment method for low speed microflows. Continuum Mech. Thermodyn. doi:10.1007/s00161-009-0128-y

26. Bennoune, M., Lemou, M., Mieussens, L.: An asymptotic preserving scheme for the Kac model of the Boltzmann equation in the diffusion limit. Continuum Mech. Thermodyn. doi:10.1007/s00161-009-0116-2

27. Seibold, B., Frank, M.: Optimal prediction for moment models: crecsendo diffusion and reordered equations. Continuum Mech. Thermodyn. doi:10.1007/s00161-009-0111-7 